\title{
Biphosphonates-related osteonecrosis of the jaw: Clinical and physiopathological considerations
}

\author{
Alberto Borgioli' \\ Christian Viviani' \\ Marco Duvina' \\ Leila Brancato' \\ Giuseppe Spinelli' \\ Maria Luisa Brandi ${ }^{2,3}$ \\ Paolo Tonelli' \\ 'Department of Odontostomatology, \\ Dental School; ${ }^{2}$ Department \\ of Internal Medicine; ${ }^{3}$ DeGene \\ Spin-off, Medical School, University \\ of Florence, Florence, Italy
}

\begin{abstract}
Since osteonecrosis of the jaw was related to biphosphonate administration by Marx, studies showing clinical symptoms, drug and surgical therapies overwhelmed the literature. Furthermore, the literature demonstrated the correlation between chronic biphosphonate adsumption and osteonecrosis of the jaw onset. Nitrogen-containing biphosphonates are widely used for the management of metastatic cancer, for prevention and treatment of osteoporosis, for the treatment of Paget's disease, and for the management of acute hypercalcemia. According to our experience, the treatment of BRON-J's lesions is difficult and prolonged. For this reason, in order to avoid these complications it is mandatory to perform a risk staging in patients who must undergo biphosphonate administration. When pharmacologic treatments with antibiotics and local antiseptics are not able to control the development of BRON-J's complications, the clinicians should perform radical surgical treatments such as the resection of the bone involved.
\end{abstract}

Keywords: osteonecrosis of the jaw, biphosphonates, BRON-J

\section{Introduction}

Osteonecrosis of the jaw is a chronic osteomielitis that recognizes a multifactorial genesis, connected to both local and systemic factors. The relevant systemic factors capable to influence development of osteonecrosis of the jaw encompass immunosuppression, chemotherapy, corticosteroid therapy, and endocrine diseases.

Pharmacologic-related osteonecrosis of the jaw (BRON-J) in oncologic patients treated with intravenous biphosphonates was an unknown clinical entity until 2003, when Marx described 36 cases of BRON-J in patients affected by malignant tumors. ${ }^{1}$ Biphosphonates stand as an important group of drugs for the treatment of metabolic and oncologic pathologies involving the skeletal system. Biphophonates act by inhibiting osteoclastic bone resorption. The most common drugs utilized in the prevention and therapy of osteoporosis are: alendronate, risendronate, ibandronate, and clodronate. Pamidronate and zolendronate are utilized in the prophylaxis of bone complications and in the hypercalcemia associated to multiple myeloma and to metastatic bone disease due to breast and prostatic cancer. All these chemical substances are characterized by a high power and selectivity. Nowadays, the literature demonstrates the correlation between chronic biphosphonate assumption and onset of osteonecrosis of the jaw.

\section{BRON-J: history and definition}

Since Marx's study other studies on BRON-J have been published. In 2004, Ruggiero and colleagues published 63 cases of BRON-J, with the majority of cases being dependent on the use of intravenous biphosphonate administration in cancer patients and only few patients treated with oral biphosphonates for osteoporosis. ${ }^{2}$

In 2005, Marx published 119 cases of BRON-J and correlated it to the type of drug used, to the invasiveness of the oral treatments, to the dose, and to the assumption 
length for a given drug. ${ }^{3}$ In the same year, Scientific Societies published the first position paper on the topic. The American Academy of Oral Medicine described clinical manifestations of these lesions, suggested potential clinical ways to prevent and to treat the affected patients. ${ }^{4}$

Several authors later reported extensive revisions of myeloma and metastatic cancer disease treated with intravenous biphosphonates, correlating the extension and evolution of this complication to the type of drug and to the length of treatment. ${ }^{5-7}$

Intravenous biphosphonates became a standard therapy for the control of complications in metastatic bone disease, such as pain, local compression, spontaneous fractures, and hypercalcemia. In several clinical studies, nitrogen-containing molecules (ibandronate, pamidronate, zolendronate) showed to be more effective in controlling manifestations of systemic malignant bone disease if compared to clodronate, with zoledronate being the most potent drug in reducing bone lesions extension and in delaying the development of the first bone metastasis. . $^{89}$

Despite the high risk of BRON-J development in oncologic patients the American Society of Clinical Oncology ${ }^{10}$ recommends the use of zoledronic acid even in patients with asintomatic metastases or disease in progress. ${ }^{11}$

In 2006, significant data emerged from an American Association of Oral and Maxillofacial Surgeons position paper that reported a consistent incidence of BRON-J, depending on prolonged biphosphonate treatment together with other related risk factors, such as tooth-alveolar bone pathological conditions of inflammatory nature. ${ }^{12}$ This is especially true for patients suffering from multiple myeloma, and breast or prostatic cancer.

The American Association of Oral and Maxillofacial Surgeons also declared a clear disease staging, from not visible oral lesions to more severe clinical pictures, such as the presence of bone sequestrum and jaw osteolytic complications, proposing different therapeutic protocols based on the stage of this pathology. ${ }^{12}$

The American Association of Oral and Maxillofacial Surgeons ${ }^{12}$ established universal criteria for the BRON-J tassonomic picture that was valid when three phenotypes were present:

1) Previous or in progress assumption of biphosphonates;

2) Exposed necrotic bone of the jaws for more than eight weeks;

3) No history of radiotherapy of the maxillofacial region.

A strict correlation between BRON-J and chronic administration of biphosphonates, with incidence ranging from $0.8 \%$ to $12 \%$ is a well recognized phenomenon. ${ }^{13}$
Until 2002, however, the incidence was less than a single case out of 10,000 treated patients ${ }^{1}$ and these data refer to patients treated with nonaminobiphosphonates, such as etidronate or clodronate, at doses used in the therapy of osteoporosis. With the introduction of aminobiphosphonates (risedronate, zoledronate, ibadronate, and aledronate) more powerful in inhibiting bone resorption and in preventing osteoporotic fractures, the incidence of this complication grew to a relevant proportion of patients, especially in these with cancer, with multiple myeloma or metastatic breast, prostatic, or kidney cancer.

As suggested by the American Society of Clinical Oncology, ${ }^{14}$ zoledronate and pamidronate show a high potency in inhibiting bone and are the chosen therapies in the treatment of the malignant disorders of the skeleton. The potency of these molecules together with their intravenous administration at high dosage in oncologic patients represent the basis for the high incidence of BRON-J in these subjects when compared to osteoporotic patients treated for the prevention of fragility fractures. ${ }^{15}$ Another potential factor that plays a role in the development of BRON-J is the affinity for the hydroxyapatite crystals by the aminobiphosphonate, with zoledronate showing the highest affinity versus other molecules of this group.

The American Association of Oral and Maxillofacial Surgeons ${ }^{12}$ pointed out to other potential risk factors for BRON-J onset, such as systemic corticosteroid therapy, smoke, alcohol, bad oral hygiene conditions, chemotherapy, radiotherapy, diabetes, and blood clot diseases. The permissive local factors are: oral surgical treatments, flogistic lesions, and an excessive pressure of the removable denture on a thin mucosa. ${ }^{16}$

There is no doubt that many factors must occur in BRON-J development, such as intravenous prolonged biphosphonate administration, and a chronic or an acute periodontitis, both responsible for medullary osteomyelitis of the jaw. ${ }^{3}$

The higher incidence in females than in males could be referred to a longer exposure to oral therapy with biphosphonates in osteoporosis and to a higher incidence of breast cancer $^{17}$ in women versus men.

From a pathological point of view, BRON-J, such as osteomyelitis, begins in the undifferentiated connective bone tissue, in the Haversian wall vasa, and in the bone marrow spaces. The process progression towards the cortical bone and the periostium leads to the concurring presence of several anatomopathologic aspects of the lesion: osteolysis associated to essudation or to weak growth of granulation tissue; osteonecrosis with slow but progressive bordering of sequestra; suppurated oral/extra-oral fistula caused by 
superinfection of necrotic tissue; absence of bone remodelling, hence bone condensation at the border of the sequestrum; and hypotrophy or atrophy following loss of the bone sequestra with low coverage of defect by soft tissues.

According to the American Association of Oral and Maxillofacial Surgeons position paper, one can stage BRON-J's patients in two groups: patients treated with aminobiphosphonates with no exposed bone segments (patients at risk of BRON-J development); and patients presenting BRON-J with exposed and necrotic bone segments.

Bone exposure for more than eight weeks can worse the clinical picture. In fact, the exposed areas located in the lower jaw and having different size (from post-extraction socket site to larger areas or multiple areas) remain asymptomatic and with no signs of flogosis. The necrotic bone areas and exposed bone cause pain due to acute inflammation of surrounding soft tissues. Mucosa is reddish, swollen, bleeding and strongly painful on light pressure. Teeth close to the involved bone are often mobile and a local reactive limphoadenopathy can be noticed. The clinician can still manage this stage of pathology with conservative procedures and medical therapy; including antibacterial agents to fight infections that involve the exposed and necrotic bone. ${ }^{12}$

Purulent debris are present in endoral abscessual cavities (if the purulent swelling is held by periostium and then by perimaxillary muscels) or in extraoral cavities called perioral phlegmon (if the osteolytic area is beyond perimaxillary muscles insertions). In the first case purulent material drains in the oral cavity. In the second case, purulent material drains in preconstituted anatomical spaces delimited by connective tissue layers of the neck. Maxillary phlegmon can involve canine or buccal spaces. Mandibular phlegmon can involve submental, submaxillary, sublingual, submandibular spaces. In this case the phlogosis exceeding these anatomical limits can spread (for contiguity or through the lymphatic system) to the secondary spaces such as pterygomandibular, lateropharyngeal, masseteric and pterigo-maxillar spaces. ${ }^{18}$

The different thickness of the cortexes justifies the earlier externalization if the pathologic process is located in the upper jaw. In the lower jaw the osteolytic damage tend to became deeper sometimes reaching the inner edge of the mandibula (Figures 1,2). The involved bone can fracture spontaneously because of its reduced elasticity. Furtheremore the purulent material can compress nerve endings, causing local paresthesia. ${ }^{19,20}$ This phase, often following the relapse of the oncologic disease and/or the antiblastic treatment associated to corticosteroid therapy is defined as "complicated phase."

This phase is characterized by purulent phlogosis, by the presence of fistulae (Figure 3), by spontaneous fractures, by compromized general physical condition with fever, and by reactive adenopathy. In this stage conservative treatments associated to prolonged antibiotic therapy can be useless

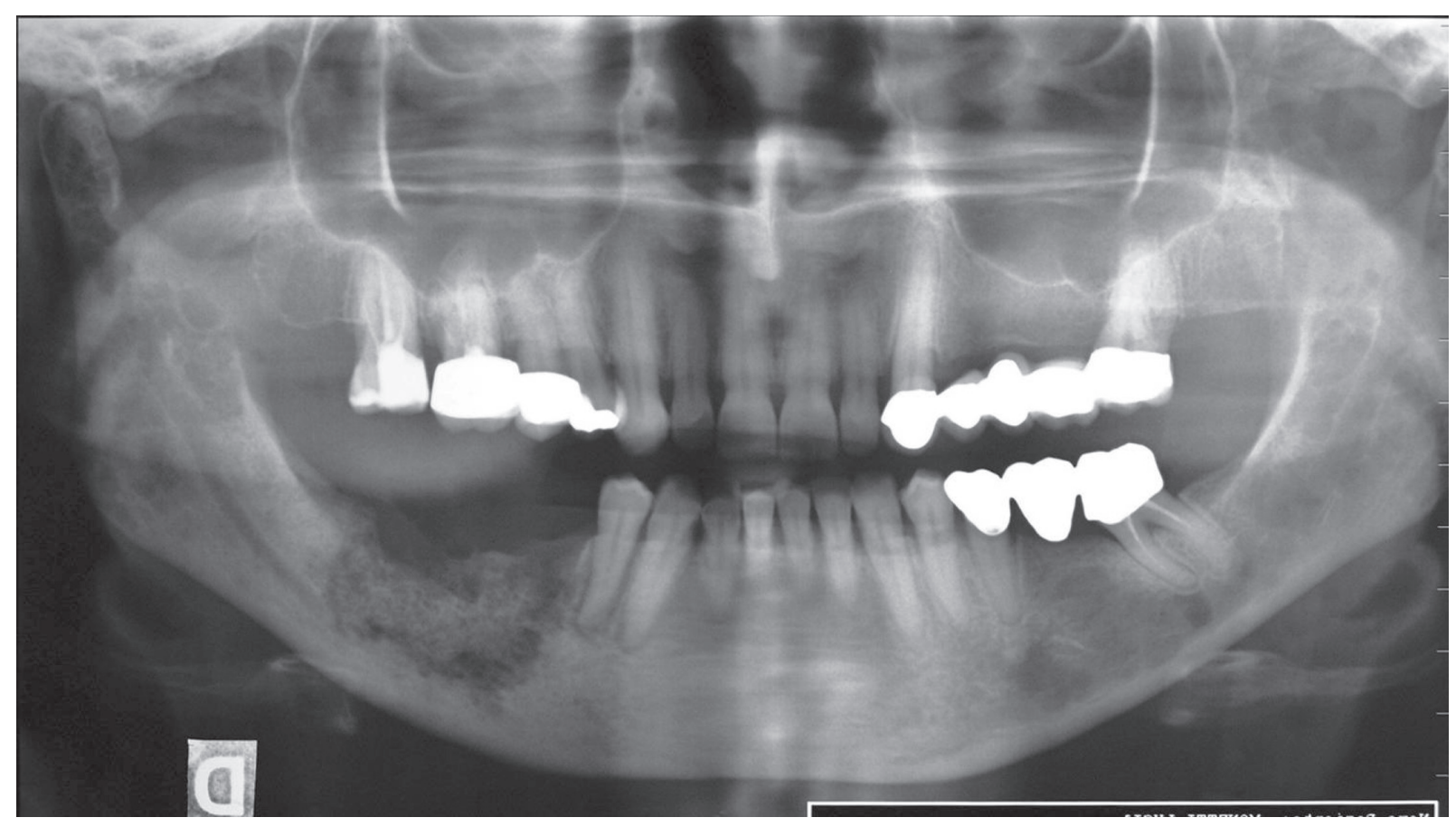

Figure I Osteolytic lesion in the lower jaw of patient treated with Zometa ${ }^{\circledR}$ for 12 months (59 years old, female, breast cancer). 


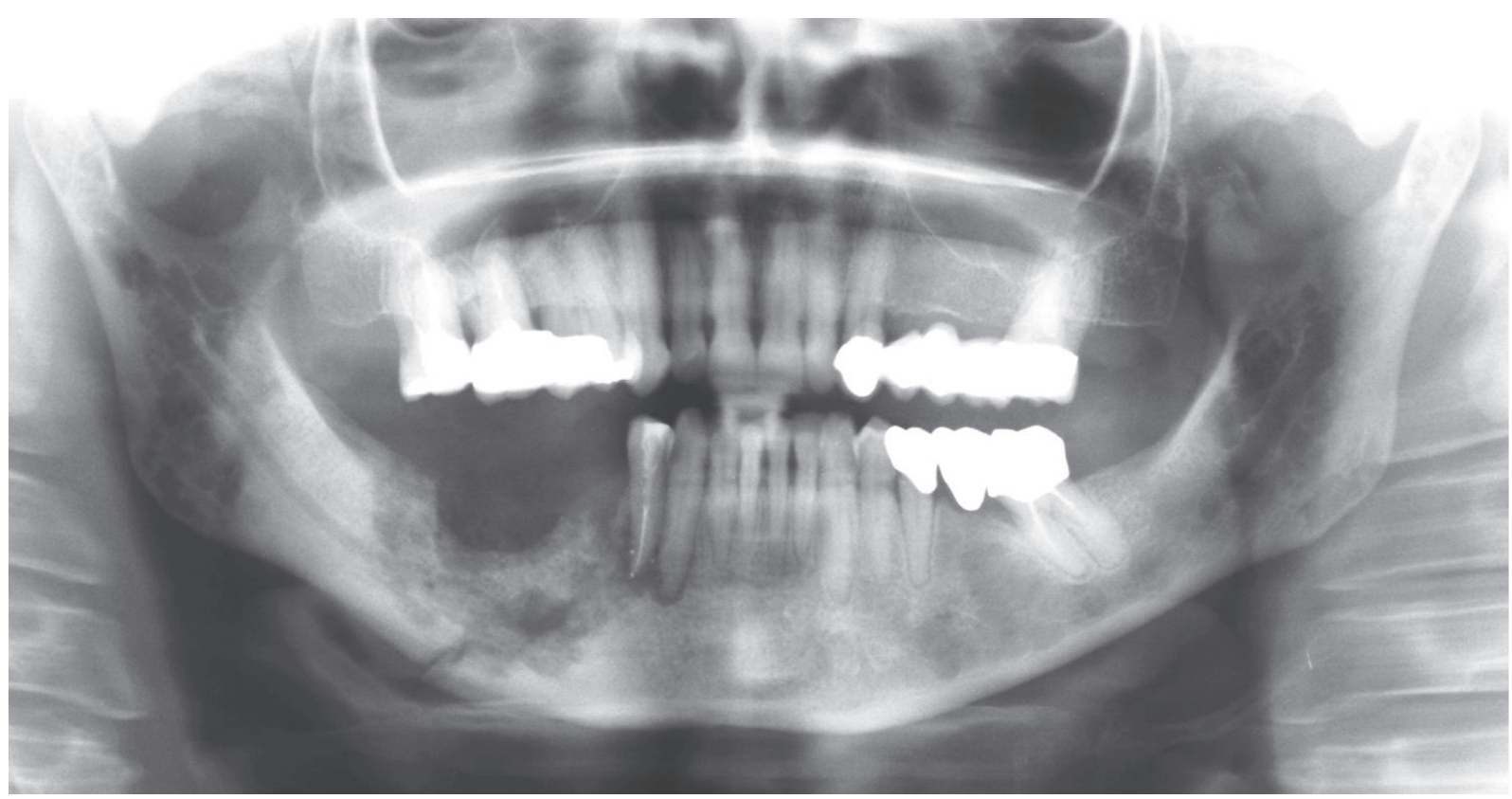

Figure 2 Worsening of the lesion after sequestrectomy in patient treated with Zometa ${ }^{\circledR}$ for 12 months (59 years old, female, breast cancer).

because of the gravity and the extension of the process, with a radical surgery being more indicated.

\section{BRON-J diagnosis}

BRON-J diagnosis is quite clear if one refers to anamnesis, natural history of the oncologic pathology and/or biphosphonates administration. The evidence of the clinical lesion is confirmed with conventional X-rays showing a radiopaque sequestrum usually rounded by diffused radiolucency with a blurred contour due to the higher mineralization of the jaw. This aspect, due to the fixation of calcium in the bone tissue, is responsible for the patchy-ragged multilocular appearance of the involved

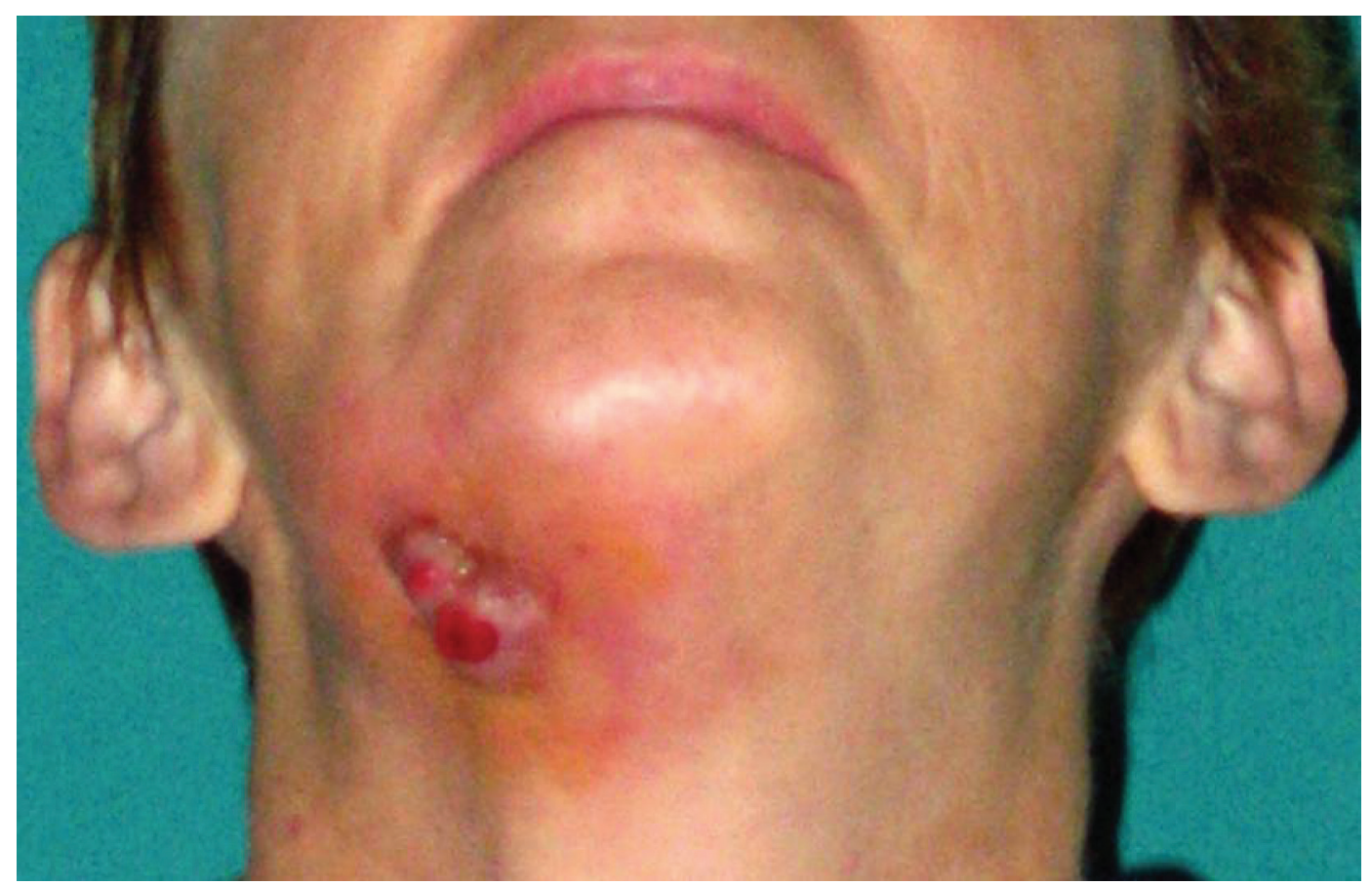

Figure 3 Cutaneous fistula in patient treated with Aredia ${ }^{\circledR}$ and Zometa ${ }^{\circledR}$ for 24 months (48 years old, female, breast cancer). 
area and it assumes a higher definition when a radiolucent osteolytic process with a central radiopaque mass of necrotic bone is identified at its periphery.

Computed tomography (CT) can help allow a higher definition of the necrotic foci and their relationships with the surrounding anatomical structures, making possible to quantify the bone sclerosis status. However, CT is not useful either in the staging of the asymptomatic patients or in the differential diagnosis between a primary tumor (with osteolytic aspect and ill-defined borders) and metastatic spreads of prostatic or breast cancer with sclerotic aspect (Figure 4). With CT it can be easier to detect mandibular myeloid lesions in high vascularized areas with their "mould", regular and well defined characteristics. In these cases the use of a contrast medium can help to better identify the lesions.

Once the sequestrum and the periosteal reactive bone deposition have been identified by CT, magnetic resonance imaging (MRI) is useful to evaluate the quality of overlying soft tissues and the medullary edema, which is a sign of ischemia and bone necrosis.

The scintigraphy (Tc99-scan) is the most sensitive diagnostic device to identify maxillary edema with vascular alterations and to localize bone necrosis even at early stages of the disease. Nevertheless this diagnostic technique has a limit: Tc99-scan is not able to make a differential diagnosis with the metastatic process. ${ }^{21,22}$

The biopsy of the bone lesions must be carefully evaluated, because the procedure itself may damage bone tissue by creating a wound which can hardly heal. ${ }^{23}$

\section{Etiopathogenesis}

Nitrogen-containing biphosphonates are used widely for the management of metastatic cancer in bone (intravenous zoledronic acid or pamidronate), for the prevention and treatment of osteoporosis (oral alendronate, risedronate, and ibandronate) for the treatment of Paget's disease of bone (intravenous pamidronate and oral aledronate and risedronate), and for the short-term management of acute hypercalcemia (intravenous zoledronic acid and pamidronate). ${ }^{24,25}$ The nitrogen moiety attached to the side chainof the middle carbon of the phosphorus-carbon-phosphorus biphosphonate backbone renders these drugs much more potent as inhibitors of bone resorption than the bisphosphonates that do not contain nitrogen (etidronate and clodronate). Bisphosphonates reduce the survival and function of osteoclasts, the bone-resorbing cells. These antiresorptive actions largely account for the

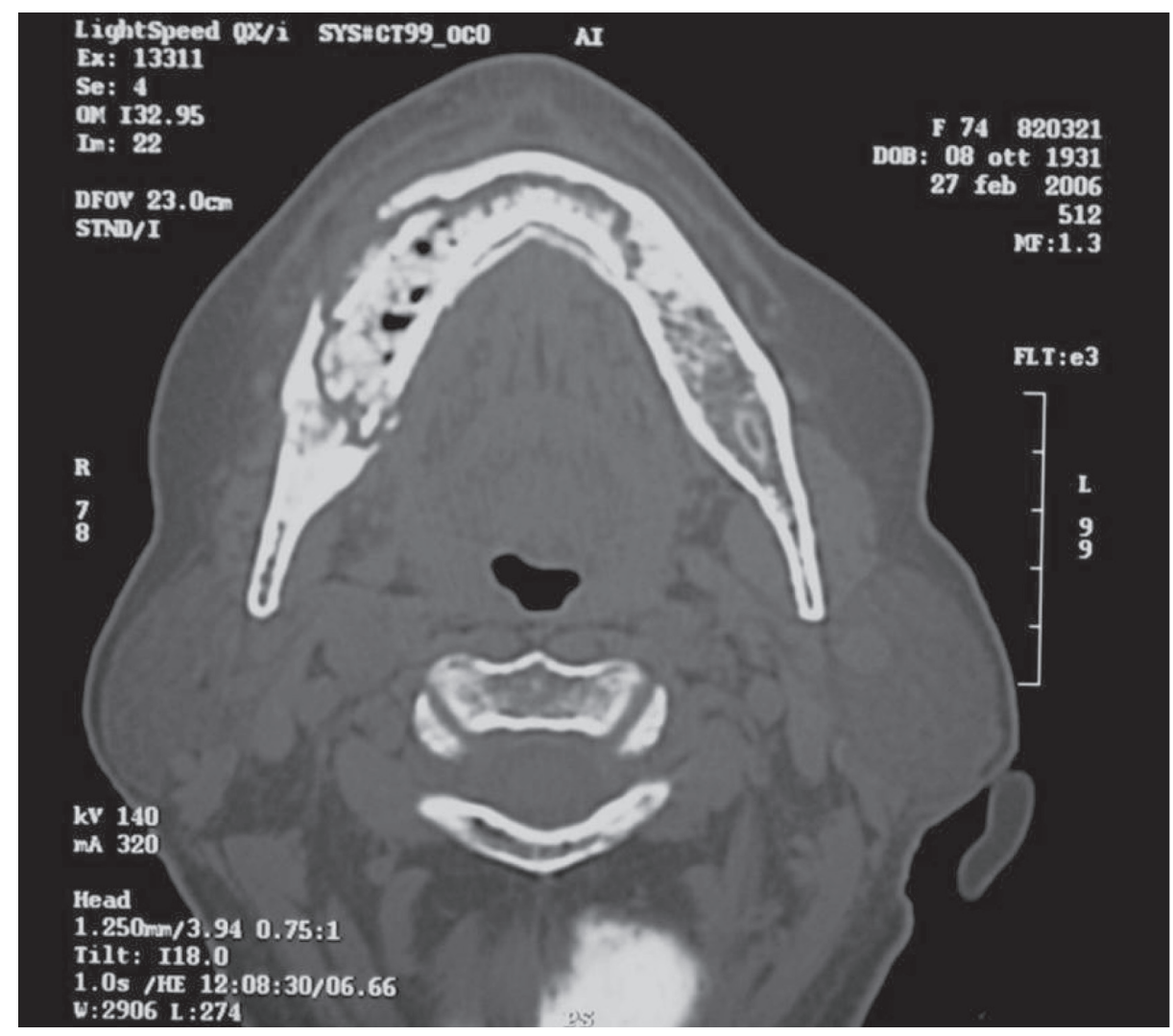

Figure 4 Computed tomographic scan of the bone sequestrum in patient treated with Zometa ${ }^{\circledR}$ for 5 months (75 years old, female, breast cancer) 
drugs'efficacy in conditions in which the rate of bone resorption exceeds the rate of bone formation.

Until recently, the only adverse events of substantial consequence associated with the nitrogen-containing bisphosphonates were upper gastrointestinal intolerance (with oral administration) and a short-lived acute phase reaction characterized by fever, myalgias, and an influenza-like syndrome (with intravenous administration). Now another potential complication of these agents-osteonecrosis of the jaw-has surfaced. ${ }^{26,27}$

\section{The Florence experience Patient's characteristics}

Based on these premises, the aim of the present study was the description of clinical and anatomopathological aspects of the disease, based on our experience in the management of BRON-J.

From February 2004 to September 2006, 19 patients (14 females and 5 males) with BRON-J undergoing intravenous biphosphonate treatments for cancer were examined at the Oral Surgery Department of the Florence University Hospital. ${ }^{28,29}$ The mean age was $66.4 \pm 11.7$.

In 14 patients the used biphosphonate was zolendronate, in one patient pamidronate and in four patients both drugs were administrated. The mean interval administration was 12 months (minimum 5 months, maximum 36 months).

In nine patients $(47.4 \%)$ the oncologic disease was breast cancer, in six patients $(31.5 \%)$ myeloma, in three patients $(15.8 \%)$ prostatic cancer, and in one patient $(5 \%)$ colon cancer. All the patients were chosen following strict diagnostic criteria. The most frequent symptoms were: spontaneous pain, swelling, odontogenic abscesses, oral fistulas, bone exposure due to mucosal ulcer, post-extraction alveolitis, and local limphoadenopathy.

The trigger factors were considered to be tooth extractions in 10 patients $(52.6 \%)$, local concussion (inadequate removable total denture, edentulous ridges) in two $(10.5 \%)$, root canal treatment in two $(10.5 \%)$, and surgery in three (15.7\%). In some cases it was not possible to identify a trigger factor. In 10 patients $(52.6 \%)$ a pre-existing inflammatory lesions appeared to worsen the development of the disease.

The patients were treated with mouth rinses (chlorhexidine gluconate $0.12 \%$ three times/daily); local or systemic antibiotic therapy (amoxicillin $1 \mathrm{~g}$ three times/daily; repeated local application of methronidazol) and, in case of mycotic overinfection, with fluconazole 200 mg/daily. Furthermore patients underwent hyperbaric treatment.
The treatment of this lesion is extremely difficult and prolonged. There are no data to support any therapeutic choice: surgery often worsens the pathology.

Surgical curettage to achieve mechanical debridement are indicated in patients with no complications. More invasive surgical treatment (such as deeper courettage, sequestrectomies, large resections, and vascularized bone grafts) are indicated after clinical changes characterized by clinical symptoms (pain, fever), oral or extra oral fistula, necrotic tissue, pathologic fractures and ineffective antibiotic treatment. In our study 13 patients out of 19 were treated with curettage and two with major surgery (segmentary mandibulectomy). Four patients were not operable.

After one-year follow-up, in most of the cases complete healing was not observed, although therapeutic protocol was strictly applied. All cases of maxillary location (two out of 19) reached complete healing thanks to secondary wound closure after two months from surgery. On the contrary we observed only symptoms of improvement in case of mandibular location, probably for the reduced regenerative capacity at this site.

In all patients pharmacological biphosphonate treatment was suspended. The interruption of biphosphonate assumption is one of the most difficult decision and should be taken in agreement with the oncologist. According to Migliorati and colleagues ${ }^{31}$ the suspension of biphosphonate treatment is mandatory, even though there is no immediate clinical improvement.

\section{Results and discussion}

The treatment of these lesions is extremely difficult and prolonged. There are no data to prefer any therapeutic choice over another, even though surgery appears to worsen the disease's course.

Surgical curettage to achieve mechanical debridement is indicated in patients without complications. Chemical debridement is carried out with antiseptic irrigations and with iodine gauze. Re-infection prevention is improved by local ointment use and $0.12 \%$ chlorhexidine daily rinses. Surgical procedures to achieve a mechanical debridement of necrotic tissue, broad spectrum antibiotic treatment for a long period, and local antibiotic use are of benefit before the progression to bone exposure and to small bone sequestra. More invasive surgical treatment (such as deeper curettage, sequestrectomies, large resections and vascularized bone grafts) are indicated in the occurrence of systemic clinical symptoms (pain, fever), of oral or extra-oral fistulas, of necrotic tissue, of pathologic fractures, and of lack of 
response to antibiotic treatment. The necrotic tissue curettage, sequestrectomy, sliding flap procedure (in two cases with oro-antral communication) and peduncle vascularized bone graft (in case of fracture) were the surgical treatments used in order to stop osteonecrotic lesion progression (Figure 5).

Metastatic foci were not shown by histological examination both in the lesion core and in the neighbouring bone tissue. Macroscopic healthy bone samples showed cortical necrosis with well preserved lamellar bone. Furthermore, empty osteocytic lacunae were detected and medullary bone tissue appeared necrotic.

All cases of maxillary location reached complete healing. In the majority of the cases of extra-maxillary location, 14 patients $(73.6 \%)$ complete healing was not achieved, although the therapeutic protocols were strictly applied. ${ }^{28,29}$ We observed only symptoms of improvement when the location was in the lower jaw: five patients $(26.3 \%)$, probably for the reduced regenerative capacity at this site.

Following the American Association of Oral and Maxillofacial Surgery's staging and treatment criteria, ${ }^{12}$ two different clinical courses have been identified: early clinical course, where a small bone sequestrum was identified (Figure 6); and late clinical course, where large neocrotic areas worsened by suppurative phlogosis were detected (Figure 7).

The present data showed a higher incidence of BRON-J in patients treated with intravenous zolendronate and pamidronate. Clinical pictures varied from a more limited osteonecrosis areas with or without suppurative phlogosis to larger osteonecrotic areas with suppurative phlogosis, jaw fractures and extra-oral fistulae (Figure 8).

In nine patients $(47.3 \%)$ we noticed a heavy odontalgia following the extraction of teeth located in the maxillary area involved by BRON-J. Before teeth extraction, the pain was referred to periodontitis involving both the involved teeth and the maxillary area close to them. The role of biphosphonates in the onset of the lesion was confirmed by the time elapsed between drug assumption and the lesions' development (about 18 months for zoledronate and about six years for pamidronate),$^{30}$ with reports of lesions initiated even after five months from the beginning of treatment. ${ }^{31,32}$ All the patients of our study underwent a drug treatment longer than six months. The length of biphosphonate treatment represents a risk factor for BRON-J along with chemotherapy, multiple myeloma, renal failure, corticosteroid treatment, anemia, hypoproteinemia, infections etc. ${ }^{30,34}$ Six patients out of 14 under chemotherapeutic and radiotherapy treatment presented larger tissue necrosis refractory to the applied therapeutic protocols.

In our study, preferential location of osteonecrotic lesions was in the lower jaw: in 14 patients $(73.6 \%)$ the location was in the mandible, in two patients $(10.6 \%)$ was both mandible and maxillary, and in three patients $(15.8 \%)$ was only maxillary. The location in the mandible seems to be explained by terminal vascularization, lower quantity of trabecular bone in the lower jaw, and more frequent microinjures due to removable denture and masticatory forces. ${ }^{3,20}$

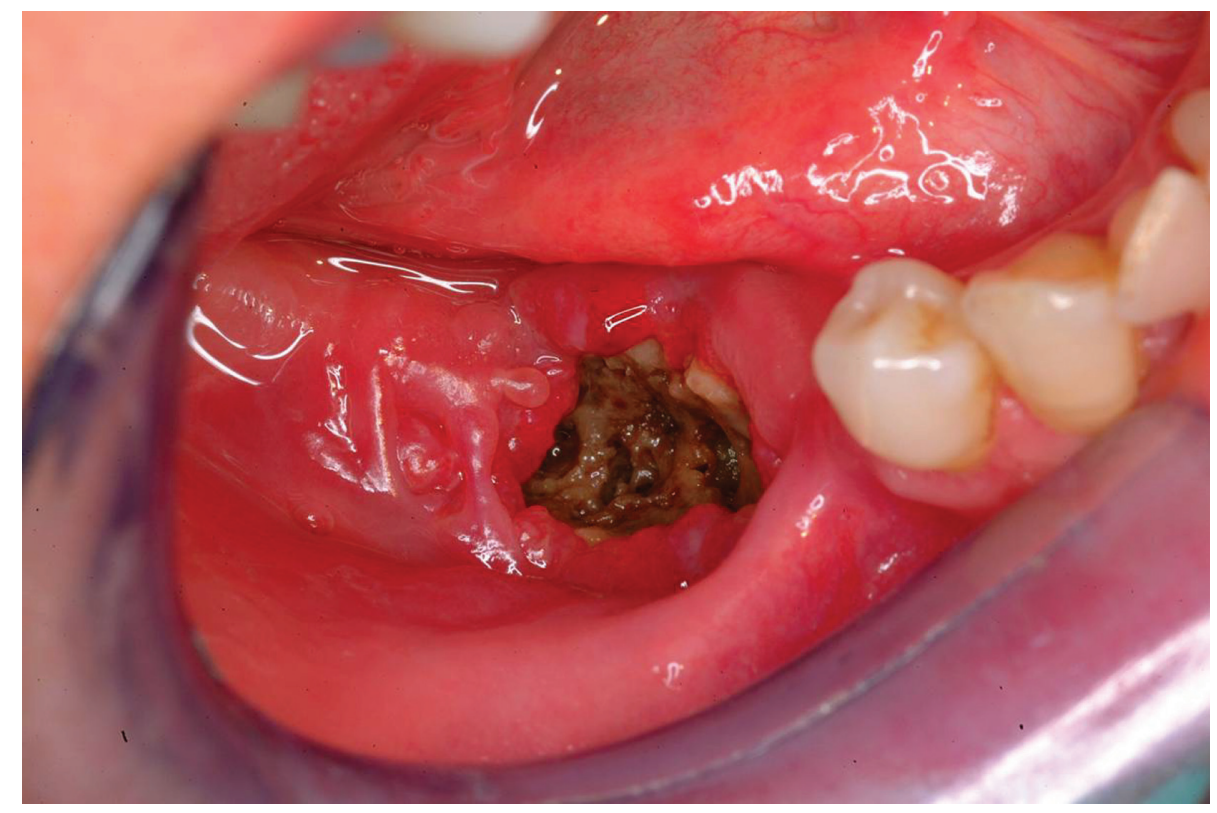

Figure 5 Spontaneous fracture of the lower jaw in patient treated with Aredia ${ }^{\circledR}$ and Zometa ${ }^{\circledR}$ for 24 months (43 years old, female, breast cancer). 


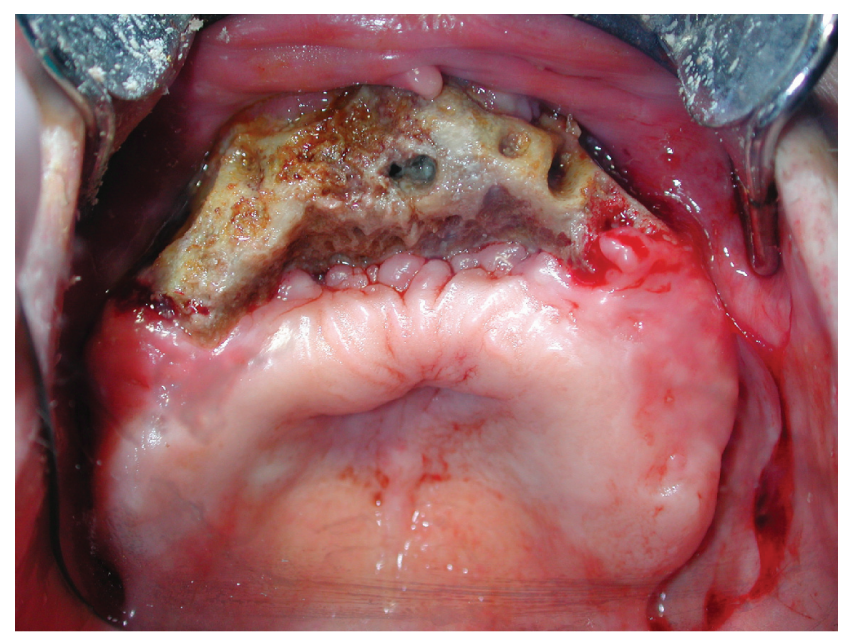

Figure 6 Early clinical picture of the lower jaw in patient treated with Aredia ${ }^{\circledR}$ and Zometa ${ }^{\circledR}$ for 24 months (48 years old, female, breast cancer).

In order to categorize patients with BRON-J, the American Association of Oral and Maxillofacial Surgeons recognized three stages of the disease. ${ }^{12}$ In stage 1 , the bone is exposed but there is no soft tissue inflammatory swelling. Sometimes there is pain before bone exposure. In stage 2 , bone is exposed with associated pain and soft tissue infection. In stage 3 , the patient is affected by the pathologic fractures, oral and extra-oral fistulae.

According to previous publications. ${ }^{16,35,36}$ and American Association of Oral and Maxillofacial Surgeons' guidelines, we treated patients in Stage 1 (five patients; 26,3\%) and Stage 2 (nine patients; 47.4\%), with small sequestra, using pharmacologic and conservative protocols. In Stage 3 (five patients; $26.3 \%$ ), when the large suppurative necrotic area did not heal, the conservative treatment led to poor results so a more invasive surgical treatment should be indicated (Table 1). ${ }^{37}$

Under a therapeutic point of view the clinician should be paid attention to the perimaxillary soft tissue study, and to their vascularization since a periostium and mucosa highly vascularized are the only possibility to try to cover the necrotic area after the removal of the sequestrum.

Oxygen therapy with a hyperbaric chamber is useful to prepare the patient to the surgical treatment and platelet-rich plasma to improve soft tissue attachment (Figure 9). ${ }^{38-42}$

\section{Conclusions}

In conclusion, BRON-J shows a complex clinical picture of unclear pathogenesis, even though it seems clearly related to intravenous biphosphonate administration. Numerous retrospective studies confirmed that pharmacologic and sur-

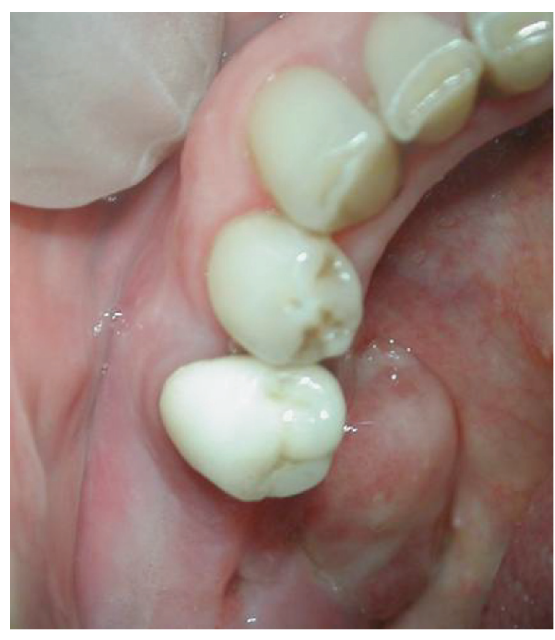

Figure 7 Advanced lesion of the upper jaw in patient treated with Zometa ${ }^{\circledR}$ for 12 months (69 years old, male, multiple myeloma).

gical therapies are not able to cure this complication, whose consequences are extremely invalidating for the patient. For this reason, several scientific societies underlined the importance of a risk staging for preventing the development of the disease in oncologic patients treated with intravenous biphosphonates as an adjuvant intervention. 4,12,23,24

In the most severe cases the treatment should guarantee: pain relief, control of the infection, prevention of the necrotic area spreading, and of the development of new contiguous lesions. When pharmacologic treatments with antibiotics and local antiseptics are not able to control the development of BRON-J's complications, the clinicians should perform radical surgical treatments, such as the resection of the bone involved followed by reconstructive surgery with

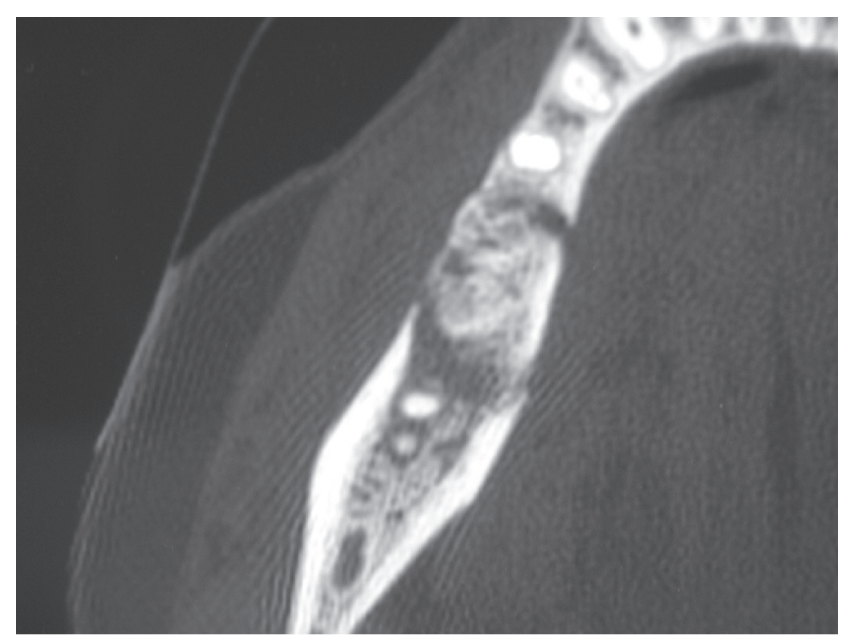

Figure 8 Abscessual complication of necrotic bone lesion in patient treated with Zometa ${ }^{\circledR}$ for II months (6I years old, male, prostatic cancer). 


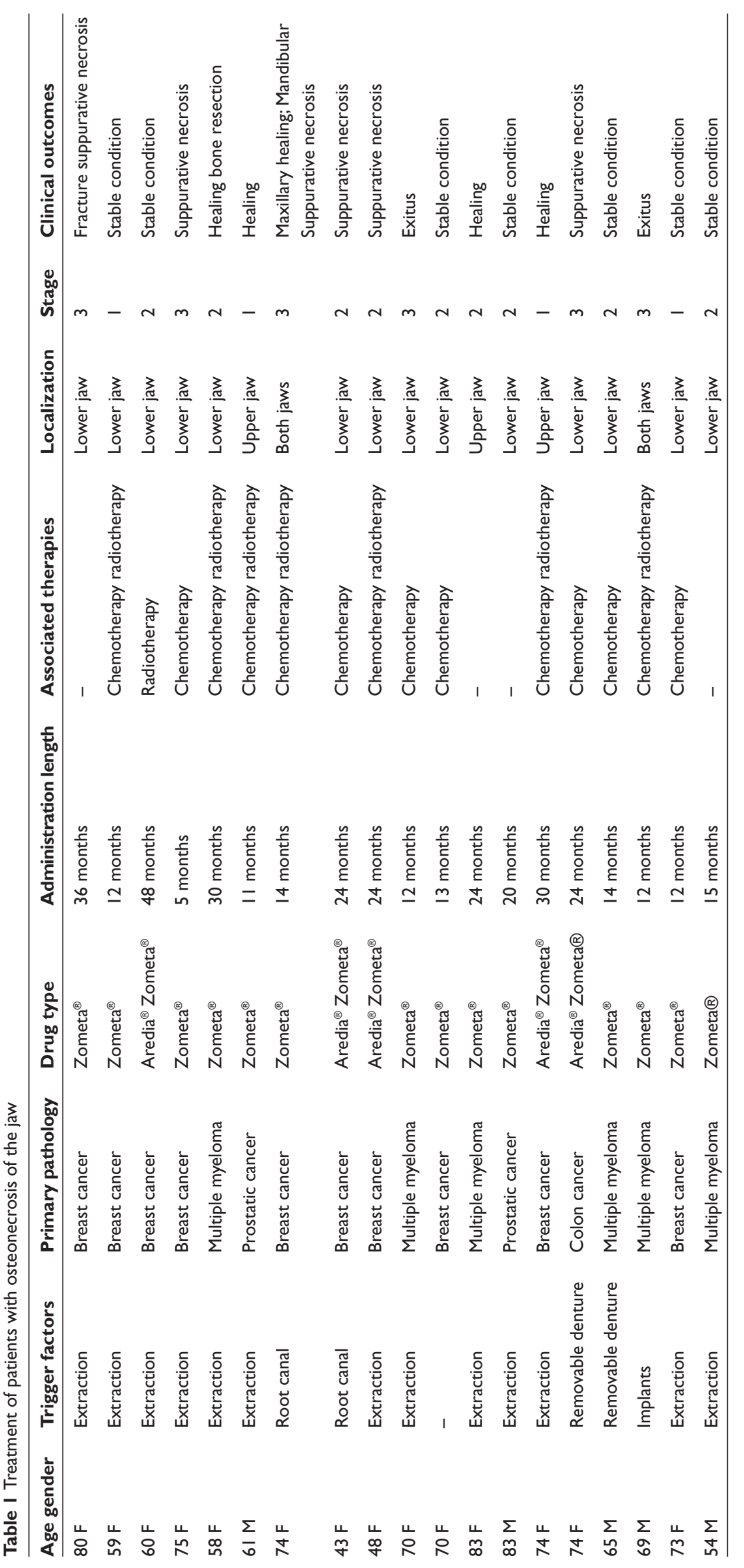




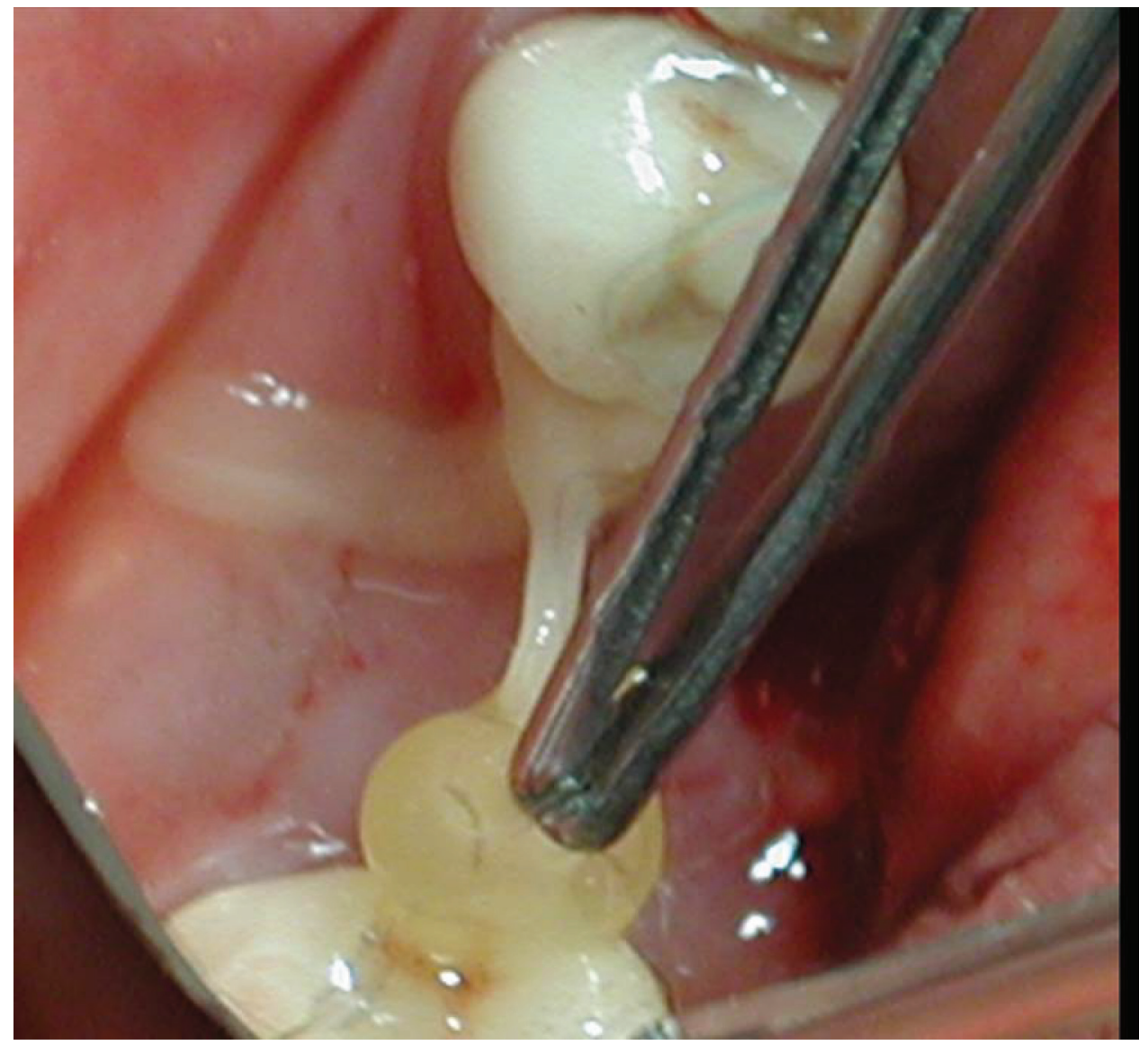

Figure 9 Use of platelet-rich plasma in the surgical treatment of the lesion in patient treated with Zometa ${ }^{\circledR}$ for 12 months (59 years old, female, breast cancer).

vascularized bone grafts. ${ }^{16}$ Today, prevention is mandatory in patients who have to be treated with biphosphonates ${ }^{43}$ and in those that are under treatment for a long period of time.

A multidisciplinary team composed by oncologists, pathologists, bone metabolism specialists, dentists, oral surgeons, and maxillofacial surgeons must cooperate to carefully evaluate the patients' clinical conditions, general and local risk factors, radiological and biohumoral exams, are useful in the prevention and in the staging of the disease.

\section{Disclosure}

The authors report no conflicts of interest in this work.

\section{References}

1. Marx R. Pamidronate (Aredia) and zoledonate (Zometa) induced avascular necrosis of the jaws: a growing epidemic. J Oral Maxillofac Surg. 2003;61:1115-1117.

2. Ruggiero SL, Mehrota B. Osteonecrosis of the jaws associated with the use of bisphophonates; a review of 63 cases. J Oral Maxillofac Surg. 2004;62:527-534.

3. Marx RE, Sawatari Y, Fortin M, Broumand V. Bisphosphonatesinduced exposed bone (osteonecrosis/osteopetrosis) of the jaws: risk factors, recognition, prevention and treatment. J Oral Maxillofac Surg. 2005;63:1567-1575.
4. Migliorati CA, Casiglia J, Epstein J, Jacobsen PL, Siegel MA, Woo SB. Managing the care of patients with bisphosphonate-associated osteonecrosis: an American Academy of Oral Medicine position paper. J Am Dent Assoc. 2005;136:1658-1668. Erratum in: J Am Dent Assoc. 2006;137:26.

5. Badros A, Weichel D. Osteonecrosis of the jaw in multiple myeloma patients: clinical features and risk factors. J Clin Oncol. 2006;24:945-952.

6. Dimopoulos MA, Kastritis E. Osteonecrosis of the jaw in patient with multiple myeloma treated with bisphosphonates: evidence of increased risk after treatment with zoledronic acid. Haematologica. 2006;91:968-971.

7. Tosi P, Zamagni E. Osteonecrosis of the jaw in newly diagnosed multiple myeloma patient treated with zoledronic acid and thalidomidedexamethasone. Blood. 2006;108:3951-3952.

8. Major PP, Cook RJ. Multiple event analysis of zoledronic acid trials in patients with cancer metastases to bone. Proc Am Soc Clin Oncol. $2003 ; 22: 762$.

9. Rosen LS, Gordon DH. Zoledronic acid is superior to pamidronate for the treatment of bone metastases in breast carcinoma patients with at least one ostelytic lesion. Cancer. 2004;100:36-43.

10. Berenson JR, Hillner BE, Kyle RA, et al. American Society of Clinical Oncology Bisphosphonates Expert Panel. American Society of Clinical Oncology clinical practice guidelines: the role of bisphosphonates in multiple myeloma. J Clin Oncol. 2002;20:3719-3736.

11. Body JJ. Breast cancer: bisphosphonates therapy for metastatic bone disease. Clin Cancer Res. 2006;12(20 Suppl):6258-6263.

12. American Association of Oral and Maxillofacial Surgeons. Position paper on bisphosphonate-related osteonecrosis of the jaws, approved by the Board of Trustees September 25, 2006 [cited on 2008 Dec 6]. Available from http://www.aaoms.org/docs/position_papers/osteonecrosis.pdf. 
13. Durie BGM, Katz M. Osteonecrosis of the jaws and bisphosphonates. N Engl J Med. 2005;353:99-102; discussion 99-102.

14. Hillner BE, Ingle JN. ASCO 2003 update on the role of Bisphosphonates and bone health issues in women with breast cancer. J Clin Oncol. 2003;21:40-42.

15. Bilezikian JP. Osteonecrosis of the jaw. Do Bisphosphonates pose a risk? N Engl J Med. 2006;355:2278-2281.

16. Ruggiero SL, Fantasia J, Carlson E. Bisphosphonate-related osteonecrosis of the jaw: background and guidelines for diagnosis, staging and management. Oral Surg Oral Med Oral Path Oral Rad Endod. 2006;102:433-441.

17. Conte PF, Guarnieri V. Safety of intravenous and oral bisphosphonates and compliance with dosing regimens. Oncologist. 2004;9(Suppl 4):28-37.

18. Peterson LJ, Ellis E. Contemporary oral and maxillofacial surgery. New York: Mosby; 1998. p. 418-432.

19. Viale PH, Lin A: Exposed bone in oral cavities. J Clin Oncol Nurs. 2005;9:355-357.

20. Ficarra G, Beninati F, Rubino I, et al. Osteonecrosis of the jaws in periodontal patients with a history of bisphosphonates treatment. JClin Periodontol. 2005;32:1125-1128.

21. Chiandussi S, Biasiotto M, Dore F, Cavalli F, Cova MA, Di Leonarda R. Clinical and diagnostic imaging of bisphosphonate-associated osteonecrosis of the jaws. Dentomaxillofac Radiol. 2006;35:236-243.

22. Hermans R, Fossion E, Ioannides C, Van de Bogaert W, Ghekiere J, Baert AL. CT findings in osteoradionecrosis of the mandibule. Skeletal Radiol. 1996;25:31-36.

23. Woo SB, Hellstein JW, Kalmar JR. Narrative [corrected] review: bisphosphonates and osteonecrosis of the jaws. Ann Intern Med. 2006;144:753-761.

24. Resza AA, Rodan GA. Nitrogen-containing bisphosphonates mechanism of action. Mini Rev Med Chem. 2004;4:711-719.

25. Green JR. Bisphosphonates preclinical review. Oncologist. 2004;9(Suppl 4):3-13.

26. Nase JB, Suzuki JB. Osteonecrosis of the jaw and oral bisphosphonate treatment. J Am Dent Assoc. 2006;137:1115-1119.

27. Vescovi P, Merigo E, Melet M, Manfredi M. Bisphosphonate-associated osteonecrosis (BON) of the jaws: a possibile treatment? J Oral Maxillofac Surg. 2006;64:1460-1462.

28. Tonelli P, Duvina M, Brancato L, Viviani C. Osteonecrosis of the jaw: A dramatic complication in patients with history of bisphosphonates treatment and bone disease. Study of 19 cases. Monaco: Poster Session in International Symposium, Osteology; May 10-12, 2007.

29. Borgioli A, Tonelli P, Brandi ML, Giombetti A, Duvina M, Spinelli G, Brancato L. Osteonecrosi dei mascellari da bifosfonati. L'Esperienza Fiorentina: Aspetti clinici e terapeutici. Alessandria: Abstract Presentation in Workshop: BRONJ, present and future, gennaio 20, 2007.
30. Durie BG. Osteonecrosis of the jaw and bisphosphonates. $N$ Engl $J$ Med. 2005;353:99-102.

31. Migliorati CA, Schubert MM, Peterson DE, Seneda LM. Bisphosphonateassociated osteonecrosis of mandibular bone. Cancer. 2005;104,1: 83-93.

32. Jimenez-SorianoY, Bagan JV. Bisphosphonates as a new cause of drug-induced jaw osteonecrosis: an update. Med Oral Patol Oral Cir Bucal. 2005;10(Suppl 2):88-91.

33. Robinson NA. Bisphosphonates a word of caution. Ann Acad Med Singapore. 2004;33:48-49.

34. Novartis Pharmaceuticals Corporation. Appendix 11: Expert panel recommendation for the prevention, diagnosis and treatment of osteonecrosis of the jaw. Oncologic Drugs Advisory Committee (ODAC), Meeting March 4, 2005 [cited 2008 Dec 6]. Available from: http://www.fda.gov/OHRMS/DOCKETS/AC/05/briefing/20054095B2_02_12-Novartis-Zometa-App-11.pdf

35. Mehrotra B, Ruggiero S. Bisphosphonates complications including osteonecrosis of the jaw. Hematology Am Soc Hematolol Educ Program. 2006;3:356-360.

36. Ruggiero SL, Gralow J, Marx RE, Hoff AO, Schubert MM, Huryn JM. Practical guidelines for the prevention, diagnosis and treatment of osteonecrosis of the jaw in patients with cancer. J Clin Oncol Prac. 2006;2:7-14.

37. Zavras AL, Zhu S. Bisphosphonates are associated with increased risk for jaw surgery in medical claims data: is it osteonecrosis? J Oral Maxillofac Surg. 2006;64:917-923.

38. Martins M, Saraceni G, Koga DH, Feber O, Olivetra dos Santos M, Zardetto C. Treatment of avascular osteonecrosis of the mandibule in cancer patients with a history of bisphosphonate therapy by combining bone resection and autologous platelet-rich plasma: report of 3 cases. J Oral Maxillofac Surg. 2007;65:349-355.

39. Shimura K, Shimazaki C, Taniguchi K, et al. Hyperbaric oxygen in addition to antibiotic therapy is effective for bisphosphonate-induced osteonecrosis of the jaw in a patient with multiple myeloma. Int $J$ Hematol. 2006;84:343-345.

40. Tonelli P, Brancato L, Paggetti B, Duvina M, Borgioli A. La terapia iperbarica nel trattamento dell'osteomielite dei mascellari. Rome: Sessione Poster Collegio dei Docenti in Odontoiatria Roma; 2004.

41. Giombetti A, Borgioli A, Brancato L, Spinelli G. L'Osteomielite farmacologica dei mascellari. Montecatini Terme: Poster Presentation, Congresso Nazionale della Società Italiana di Chirurgia Orale (SICO): la Pianificazione del trattamento in Chirurgia Orale; Ottobre 7-8, 2005.

42. Borgioli A, Duvina M, Brancato L, Duvina G, Tonelli P. Bad and good bisphosphonates in implantology: Clinical report. Rome: Sessione Poster Collegio dei Docenti in Odontoiatria Roma; 2007.

43. Mavrokokki T, Cheng A, Stein B, Goss A. Nature and frequency of bisphosphonate-associated osteonecrosis of the jaws in Australia. J Oral Maxillofac Surg. 2007;65:415-423. 
